\title{
Millimeter-scale gate-tunable graphene nanoribbon devices as a platform for mid-infrared and bio sensing applications
}

\author{
Akhilesh K Singh, ${ }^{1,2 *}$ Yung-Chen Lin, ${ }^{1}$ Chris J Sheehan, ${ }^{1}$ Andrew M. Dattelbaum ${ }^{2}$, Gautam \\ Gupta, ${ }^{2}$ Aditya D Mohite ${ }^{2}$ \\ ${ }^{1}$ Center for Integrated Nanotechnologies, Los Alamos National Laboratory, Los Alamos, New Mexico, 87545, USA \\ ${ }^{2}$ MPA-11 Materials Synthesis and Integrated Devices, Los Alamos National Laboratory, Los Alamos, New Mexico \\ 87545, USA \\ *Email: akhilesh@lanl.gov
}

\begin{abstract}
:
The experimental observation of graphene plasmon resonance has generated tremendous interest due to numerous potential applications such as photosensors, detectors, biosensors, and switches from $\mathrm{THz}$ to mid-infrared regime. However, practical applications require much larger dimensions ( $\mathrm{mm}$ to $\mathrm{cm}$ scale) than that demonstrated in the proof-of-concept devices. Moreover, such devices also require a detailed understanding of ribbon-to-ribbon interaction, which has not been investigated so far. Here we demonstrate gate tunable plasmon resonance in the midinfrared spectral region on millimeter-scale graphene nanoribbon (GNR) array devices fabricated using graphene monolayer. Gate dependent Fourier-transform infrared (FTIR) transmission measurements on GNR of various widths were investigated experimentally. The shift in plasmon resonance peaks of wave number $100 \mathrm{~cm}^{-1}$ at applied external gate voltage $-100 \mathrm{~V}$ was observed. This shift is attributed to strong gate modulation. Our investigation of ribbon-to-ribbon interaction by tuning the aspect ratio reveals strong modulation of surface plasmon resonance peaks in GNR. This suggests that plasmon resonances are coupled as evidenced by blue-shifted
\end{abstract}


plasmon resonance. These studies demonstrate that large-area GNR devices can serve as an ideal platform for ultrasensitive sensing and detector applications.

\section{Introduction}

Graphene plasmonics has attracted huge interest due to its unique tunability and potential applications in nano-plasmonic devices [1-15]. Graphene plasmons have been predicted and experimentally observed in the terahertz $[3,7,11,12]$ to mid-infrared $[10,13]$ region by fabricating graphene nanoribbons of specific dimensions. In the mid-infrared regime, multiple plasmon resonances have been observed, each associated with a specific physical origin. Some of these resonances are due to interaction between substrate optical phonons and intrinsic graphene plasmons, whereas other resonances are caused by light-graphene plasmon coupling in mid-infrared region. The energy of these plasmon resonance frequencies can be tuned by varying carrier density using an electrostatic gate field or by adjusting geometrical aspect ratio of the GNR. Here the aspect ratio $L / W$ is defined as the ratio of length $L$ (ribbon width $W$ plus spacing between ribbons $S$ ) to the width of GNR (see Fig. 4(a)). This offers an opportunity for using graphene based plasmonic devices as sensors, detectors, switches and other optoelectronic devices [3, 7, 10, 13-15]. While the proof-of-concept graphene plasmonic devices have been demonstrated, typical device dimensions have been limited to $80 \times 80 \mu \mathrm{m}^{2}$, inspite of the ability to synthesize high quality large-area graphene using chemical vapor deposition (CVD). Moreover, understanding the role of ribbon-ribbon interaction by varying the aspect ratio of GNR remains widely unexplored and is a key factor to precisely controlling and operating such plasmonic devices. So far, studies aimed at understanding ribbon-ribbon interactions have been limited in scope [15-18]. Recently, Yang et al. investigated plasmon hybridization in coplanar graphene nanoribbons in light of inter-ribbon spacing and carrier density using IR spectroscopy 
[18]. They found that coupling strength between nanoribbons was mediated by substrate phonons and is nearly independent of the width of nanoribbons. While these are instructive studies, the evidence for ribbon-ribbon interaction in the field of graphene plasmonics remains an open question.

In this paper, we report the experimental study of tunable plasmon excitations and lightplasmon coupling on millimeter-scale GNR array devices. GNR array devices of various widths were fabricated and defined using electron beam lithography. We demonstrate that plasmon resonance can be tuned by changing the width of GNR, applied gate voltage, and the aspect ratio of GNR arrays in the mid-IR frequency range. The gate tunability of plasmon resonance peaks persists in graphene ribbons of various widths ranging from $50 \mathrm{~nm}$ to $500 \mathrm{~nm}$. Furthermore, we studied the ribbon-ribbon interaction by tuning the aspect ratio of GNR. Here we study GNR of width $150 \mathrm{~nm}$ using various aspect ratios ranging from 1.5 to 4 . We demonstrate that surface plasmon resonance can be modulated by tuning the aspect ratio of GNR. The oscillating dipole electric field in a GNR protrudes from the edge of the GNR and effectively interacts with nearby ribbons as evidenced by blue shift in plasmon peaks.

\section{Experimental}

Here we study plasmon resonance behavior of GNR using polarization and gate dependent FTIR on back-gated GNR array devices. The GNR arrays are fabricated from monolayer graphene grown using $\mathrm{CVD}$ [19] and transferred onto $\mathrm{Si} / \mathrm{SiO}_{2}$ (intrinsic) substrate. Electron beam lithography and oxygen plasma etching were used to pattern $700 \times 700 \mu \mathrm{m}^{2}$ and $500 \times 500$

$\mu \mathrm{m}^{2}$ GNR array devices. The pattern was etched into the graphene nanoribbon using oxygen plasma at 300 mTorr and $45 \mathrm{~W}$ for $35 \mathrm{~s}$. Source and drain electrodes were defined on GNR array devices by a physical shadow mask and Ti/Au deposition using electron beam evaporation while 
the back-gate contact was made using Ag paint on Si. Electrical measurements were carried out using a B1500A semiconductor parameter analyzer. Gate dependent IR transmittance spectra of the GNR array devices are obtained using FTIR (Bruker Vertex 80) combined with an infrared microscope (Bruker Hyperion) equipped with a $15 \times$ objective and a liquid $\mathrm{N}_{2}$-cooled mercury cadmium telluride detector. The polarization of the incident light from IR source was controlled using an IR polarizer.

\section{Results and discussion}

Gate and polarization dependent FTIR measurements were performed on GNR array devices of various ribbon widths. Graphene plasmons can only be excited when electric field-vector of light is perpendicular to the length axis of GNR. The presence of the ribbon breaks the symmetry and provides necessary momentum to the dipole along the GNR width. Figure 1(a) and 1(b) shows a $7 \times 7$ array of $100 \mu \mathrm{m}$ patterned GNR array devices and a typical SEM image of GNR of width $100 \mathrm{~nm}$. Figure 1(c) shows a typical gate dependent measurement of the GNR device. As seen from Fig. 1(c), the Dirac point is close to $+80 \mathrm{~V}$, which corresponds to Fermi level $(0.4 \mathrm{eV})$ below the intrinsic Fermi level.

Figure 2(a) illustrates transmission spectra as a function of wavenumber for different gate voltages ranging from $-100 \mathrm{~V}$ to $+100 \mathrm{~V}$ for ribbons of width $100 \mathrm{~nm}$. The spectra obtained are normalized at back-gate voltage of $+100 \mathrm{~V}$ considering the fact that the charge neutrality point is close to the bias voltage. Figure 2(a) shows the full spectrum wavenumbers taken from 600 $7000 \mathrm{~cm}^{-1}$ covering all the plasmon resonance peaks along with IR absorption. At $V_{g}=-100 \mathrm{~V}$, maximum IR transmission decreases with increase in $V_{g}$. Fig. 2(b) illustrates several plasmon resonance peaks in the region $600-2000 \mathrm{~cm}^{-1}$. 
The position of these peaks is found to be consistent with previous reports $[10,13,20]$. Yan et al. investigated the tunability of surface plasmon resonances as a function of ribbon size [10]. We demonstrate the tunability of surface plasmon with gate bias voltage as well as ribbon width on a millimeter-scale GNR array device. The plasmon peaks for the GNR of width $100 \mathrm{~nm}$ at gate voltage $-100 \mathrm{~V}$ are $\mathrm{P}_{1} \sim 794, \mathrm{P}_{2} \sim 950$, and $\mathrm{P}_{3} \sim 1400 \mathrm{~cm}^{-1}$. The plasmon peaks $\mathrm{P}_{1}$ and $\mathrm{P}_{2}$ result from the coupling of graphene plasmons and substrate optical phonons $[10,13]$ often referred to as surface plasmon phonon polaritons (SPP). The plasmon resonance peak $\mathrm{P}_{3}$ is the graphene plasmon (GP). The linewidth of peak $\mathrm{P}_{3}$ is broader in comparison to peaks $\mathrm{P}_{1}$ and $\mathrm{P}_{2}$. The peaks $\mathrm{P}_{1}$ and $\mathrm{P}_{2}$ are affected by $\mathrm{SiO}_{2}$ phonon damping effects. The plasmon resonance peaks at 950 and $1400 \mathrm{~cm}^{-1}$ are strongly gate tunable as illustrated in Fig. 2(b). Both peaks $\mathrm{P}_{2}$ and $\mathrm{P}_{3}$ are blue shifted and the strength of oscillation increases with increase in applied gate voltage. The line width of the peaks also increases with increasing gate voltage. There is a shift of roughly 60 and $100 \mathrm{~cm}^{-1}$ in peak positions $\mathrm{P}_{2}$ and $\mathrm{P}_{3}$ respectively as shown in Fig. 3(a). As we vary the gate voltage from +100 to $-100 \mathrm{~V}$, more carriers are added to the graphene sheet. The shift in plasmon resonance peaks with applied external gate voltage is observed up to $100 \mathrm{~cm}^{-1}$ in GNR of width $100 \mathrm{~nm}$.

Figure 3(b) illustrates the experimental plasmon dispersion of GNR of widths ranging from 50 to $500 \mathrm{~nm}$. The dispersion relation in Fig. 3(b) predicts that the plasmon frequency $\omega_{p}$ depends on width $W$ as $\omega_{p} \propto(1 / \sqrt{W})$. All the plasmon resonance peaks $\left(\mathrm{P}_{1}, \mathrm{P}_{2}\right.$, and $\left.\mathrm{P}_{3}\right)$ are blue shifted with decrease in ribbon width. These peaks follow the same trend as discussed in the previous section for GNRs of width $100 \mathrm{~nm}$ in terms of peak intensity and peak shift with applied gate voltage. 
We further investigate the effects of ribbon-ribbon interaction and its plasmonic response for GNR of width $150 \mathrm{~nm}$ for aspect ratio ranging from 1.5 to 4 . GNR array devices with dimensions $(S-W)$ : 75-150, 300-150, and 450-150 $\mathrm{nm}$ were also investigated in detail. The aspect ratio $\mathrm{L} / \mathrm{W}$ is defined as the ratio of length $\mathrm{L}(\mathrm{L}=\mathrm{W}+\mathrm{S})$ to the width of a GNR as shown in fig. 4(a), where $\mathrm{W}$ is the width of a ribbon and $\mathrm{S}$ is space between ribbons. In each device, the width of GNR was fixed at $150 \mathrm{~nm}$ varying aspect ratios from 1.5 to 4 . Figure 4(b) shows the comparison of three different $500 \times 500 \mu \mathrm{m}^{2}$ GNR array devices on the same chip for different aspect ratios. Transmittance spectra of each pattern were obtained at background voltage +100 V. Figure 4(b) shows the FTIR transmission spectra of dimensions $(S-W)$ : 75-150, 300-150, and 450-150 nm patterns. All three plasmon peaks observed are blue shifted with increasing aspect ratios. The plasmon peaks are blue shifted by $\sim 80 \mathrm{~cm}^{-1}$ for $\mathrm{P}_{1}$, by $\sim 30 \mathrm{~cm}^{-1}$ for $\mathrm{P}_{2}$, and by $\sim 46$ $\mathrm{cm}^{-1}$ for $\mathrm{P}_{3}$ with the increase in aspect ratios from 1.5 to 4 . The surface plasmon resonances in a GNR array device are considered only from the isolated dipole in a GNR so far. Here, we consider that surface plasmon in GNR array device is due to (a) the isolated dipole in a GNR (b) the oscillating dipole electric field in a GNR protrudes from the edge of the GNR. Coupling between GNR can be thought of electric field beyond edge of a GNR, which results shift in plasmon resonance frequency upon consideration of aspect ratios. We hypothesize that the plasmon wave vector q does not simply depend on ribbon width but also on the spacing between the ribbons for the localized surface plasmons in GNR. The ribbon-ribbon interaction was not taken into account in previous studies [10, 21]. However, the weak coupling between ribbons was previously reported $[18,22]$. To understand this better, we consider the boundary conditions $[13,23]: 2 \boldsymbol{q}_{\mathrm{n}} W+2 \boldsymbol{\Phi}_{\mathrm{n}}=2 n \pi$, where $\boldsymbol{q}_{\mathrm{n}}$ is the graphene plasmon wave vector corresponding to the $\mathrm{n}^{\text {th }}$ mode, and $W$ is the width of GNR, $\boldsymbol{\Phi}_{\mathrm{n}}$ is an extra reflection phase of a surface plasmon 
propagating outside a GNR. This extra reflection phase depends on mode $\mathrm{n}$ and aspect ratio of a GNR and can be taken into account from the fact that oscillating electric field protrudes from the edge of a GNR and interacts effectively with nearby ribbons. As the aspect ratio increases, extra reflection phase decreases in accordance with the aforementioned boundary conditions. For GNR of width $150 \mathrm{~nm}$, the values of extra reflection phase factor for aspect ratios 4,3 , and 1.5 are $0.885,1$, and 1.297 respectively, as estimated from table 1 of ref. 23 . Using the extra reflection phase factor and plasmon resonance frequency from the experiment, the dispersion of plasmon resonace peaks in GNR arrays can be obtained as shown in Fig. 4(c). Furthermore, Fig. 4(b) illustrates the plasmon wave vector shift to higher frequencies with a shift of $\sim 0.27 \times 10^{5} \mathrm{~cm}^{-1}$ for GNR of width $150 \mathrm{~nm}$ as the aspect ratios increases from 1.5 to 4 . This accounts for the increase in plasmon resonance peak frequencies in GNRs for larger aspect ratios.

\section{Conclusions}

In summary, we have experimentally demonstrated that multiple plasmon resonance peaks of different widths ranging from 50 to $500 \mathrm{~nm}$ can be tuned by changing the width, applied gate voltage, and the aspect ratio of GNR in the mid-IR frequency range on millimeter-scale GNR array devices. The plasmon resonance peaks observed between $0.08-0.2 \mathrm{eV}$ are strongly gate dependent. Plasmon resonance peaks can be tuned up to wave number $100 \mathrm{~cm}^{-1}$ with a gate voltage of $-100 \mathrm{~V}$. The graphene plasmon wave vector not only depends on the width of GNR but also on the spacing between GNR. We have also studied ribbon-ribbon interaction and found that plasmon resonances are considerably blue shifted with increase in aspect ratio, keeping the width of GNR fixed. In other words, plasmon frequency is red-shifted as the spacing between ribbons decreases. This study demonstrates the fact that surface plasmon resonance peaks in GNR are due to both dipole resonances within each isolated ribbon and ribbon-ribbon coupling. 
A detailed study of ribbon-ribbon coupling in GNR will be a key factor in designing photosensors, detectors, biosensors and other optoelectronics devices ranging from $\mathrm{THz}$ to midIR region.

\section{Acknowledgements}

We would like to thank Dr. Kirill A. Velizhanin from the Theoretical Division at Los Alamos National Laboratory for useful discussions. We would also like to thank John K. Baldwin from the Center for Integrated Nanotechnologies (CINT) at Los Alamos National Laboratory for his help in metal deposition for device fabrication. This work was performed at CINT, a U.S. Department of Energy, and Office of Basic Energy Sciences user facility. The research was funded in part by the Laboratory Directed Research and Development Program and by CINT at LANL, an affirmative action equal opportunity employer operated by Los Alamos National Security, LLC, for the National Nuclear Security Administration of the U.S Department of Energy. 


\section{References:}

[1] F.H.L. Koppens, D.E. Chang, F.J. García de Abajo, Nano Letters, 11 (2011) 3370-3377.

[2] A. Vakil, N. Engheta, Science, 332 (2011) 1291-1294.

[3] L. Ju, B. Geng, J. Horng, C. Girit, M. Martin, Z. Hao, H.A. Bechtel, X. Liang, A. Zettl, Y.R. Shen, F. Wang, Nat Nano, 6 (2011) 630-634.

[4] Z. Fei, G.O. Andreev, W. Bao, L.M. Zhang, A. S. McLeod, C. Wang, M.K. Stewart, Z. Zhao, G.

Dominguez, M. Thiemens, M.M. Fogler, M.J. Tauber, A.H. Castro-Neto, C.N. Lau, F. Keilmann, D.N. Basov, Nano Letters, 11 (2011) 4701-4705.

[5] Z. Fei, A.S. Rodin, G.O. Andreev, W. Bao, A.S. McLeod, M. Wagner, L.M. Zhang, Z. Zhao, M. Thiemens, G. Dominguez, M.M. Fogler, A.H.C. Neto, C.N. Lau, F. Keilmann, D.N. Basov, Nature, 487 (2012) 82-85.

[6] J. Chen, M. Badioli, P. Alonso-Gonzalez, S. Thongrattanasiri, F. Huth, J. Osmond, M. Spasenovic, A.

Centeno, A. Pesquera, P. Godignon, A. Zurutuza Elorza, N. Camara, F.J.G. de Abajo, R. Hillenbrand, F.H.L. Koppens, Nature, 487 (2012) 77-81.

[7] H. Yan, X. Li, B. Chandra, G. Tulevski, Y. Wu, M. Freitag, W. Zhu, P. Avouris, F. Xia, Nat Nano, 7 (2012) 330-334.

[8] I. Crassee, M. Orlita, M. Potemski, A.L. Walter, M. Ostler, T. Seyller, I. Gaponenko, J. Chen, A.B.

Kuzmenko, Nano Letters, 12 (2012) 2470-2474.

[9] A.N. Grigorenko, M. Polini, K.S. Novoselov, Nat Photon, 6 (2012) 749-758.

[10] H. Yan, T. Low, W. Zhu, Y. Wu, M. Freitag, X. Li, F. Guinea, P. Avouris, F. Xia, Nat Photon, 7 (2013) 394-399.

[11] O. Mitrofanov, W. Yu, R.J. Thompson, Y. Jiang, I. Brener, W. Pan, C. Berger, W.A. de Heer, Z. Jiang, Applied Physics Letters, 103 (2013) -.

[12] H.G. Yan, Z.Q. Li, X.S. Li, W.J. Zhu, P. Avouris, F.N. Xia, Nano Letters, 12 (2012) 3766-3771.

[13] V.W. Brar, M.S. Jang, M. Sherrott, J.J. Lopez, H.A. Atwater, Nano Letters, 13 (2013) 2541-2547.

[14] Z.Q. Li, E.A. Henriksen, Z. Jiang, Z. Hao, M.C. Martin, P. Kim, H.L. Stormer, D.N. Basov, Nat Phys, 4 (2008) 532-535.

[15] J.H. Strait, P. Nene, W.-M. Chan, C. Manolatou, S. Tiwari, F. Rana, J.W. Kevek, P.L. McEuen, Physical Review B, 87 (2013) 241410.

[16] J. Christensen, A. Manjavacas, S. Thongrattanasiri, F.H.L. Koppens, F.J. García de Abajo, ACS Nano, 6 (2012) 431-440.

[17] A.Y. Nikitin, T. Low, L. Martin-Moreno, Physical Review B, 90 (2014) 041407.

[18] X. Yang, X.-T. Kong, B. Bai, Z. Li, H. Hu, X. Qiu, Q. Dai, Small, 11 (2015) 591-596.

[19] X. Li, W. Cai, J. An, S. Kim, J. Nah, D. Yang, R. Piner, A. Velamakanni, I. Jung, E. Tutuc, S.K. Banerjee, L. Colombo, R.S. Ruoff, Science, 324 (2009) 1312-1314.

[20] I.J. Luxmoore, C.H. Gan, P.Q. Liu, F. Valmorra, P. Li, J. Faist, G.R. Nash, ACS Photonics, (2014).

[21] S.A. Mikhailov, N.A. Savostianova, Physical Review B, 71 (2005) 035320.

[22] A.Y. Nikitin, F. Guinea, F.J. Garcia-Vidal, L. Martin-Moreno, Physical Review B, 85 (2012) 081405.

[23] K.A. Velizhanin, Physical Review B, 91 (2015) 125429. 


\section{Figures}

(a)

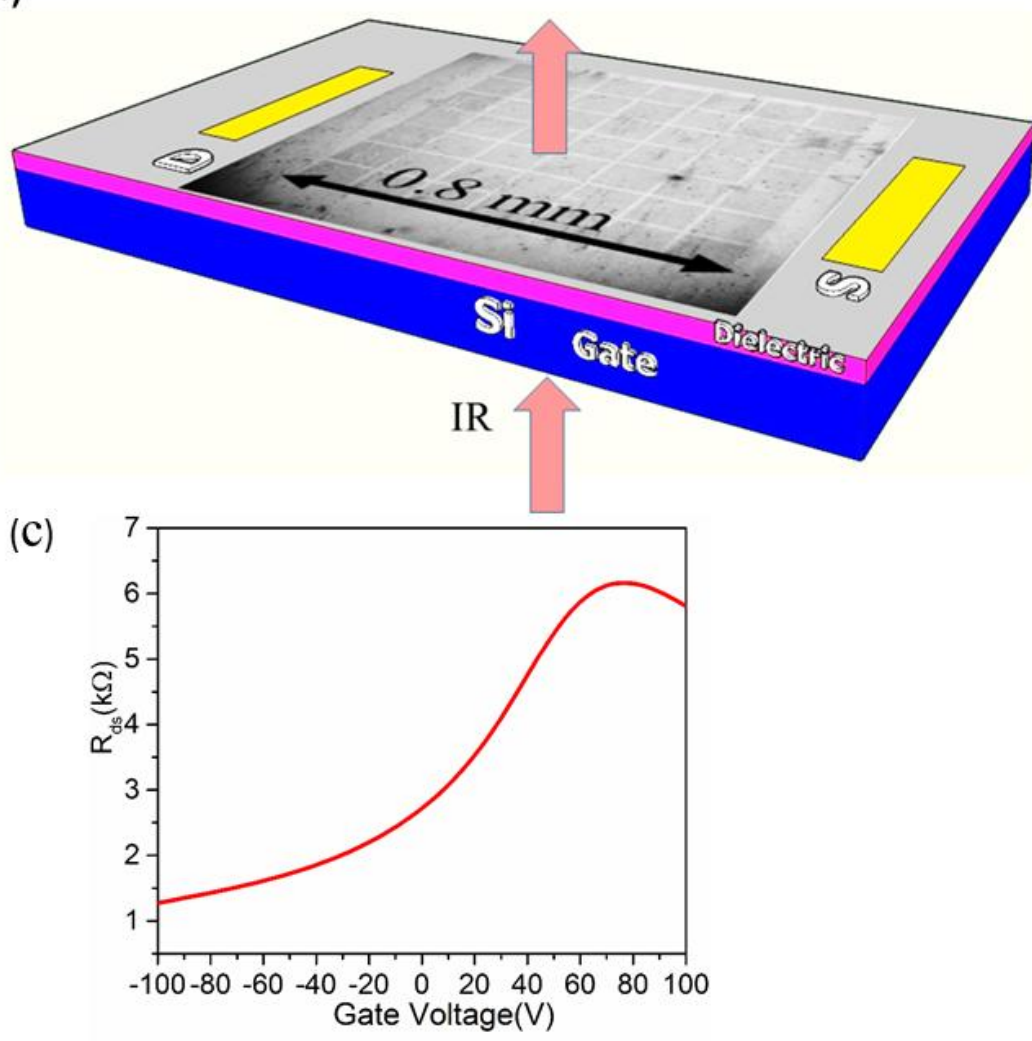

(b)

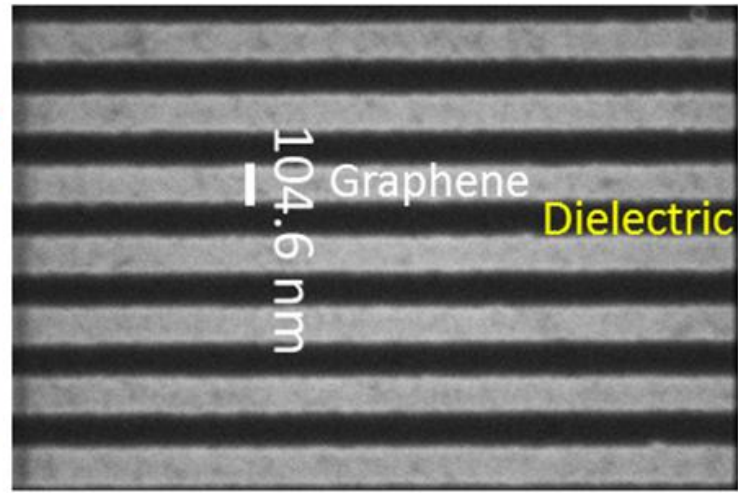

FIG.1. Schematics of the experimental device. (a) SEM image of a $7 \times 7$ array of each $100 \mu \mathrm{m}$ patterned of GNR array devices for FTIR transmission measurements (b) SEM image of GNR of width $100 \mathrm{~nm}$. (c) Electrical transport measurement of GNR array devices at ambient condition. 
(a)

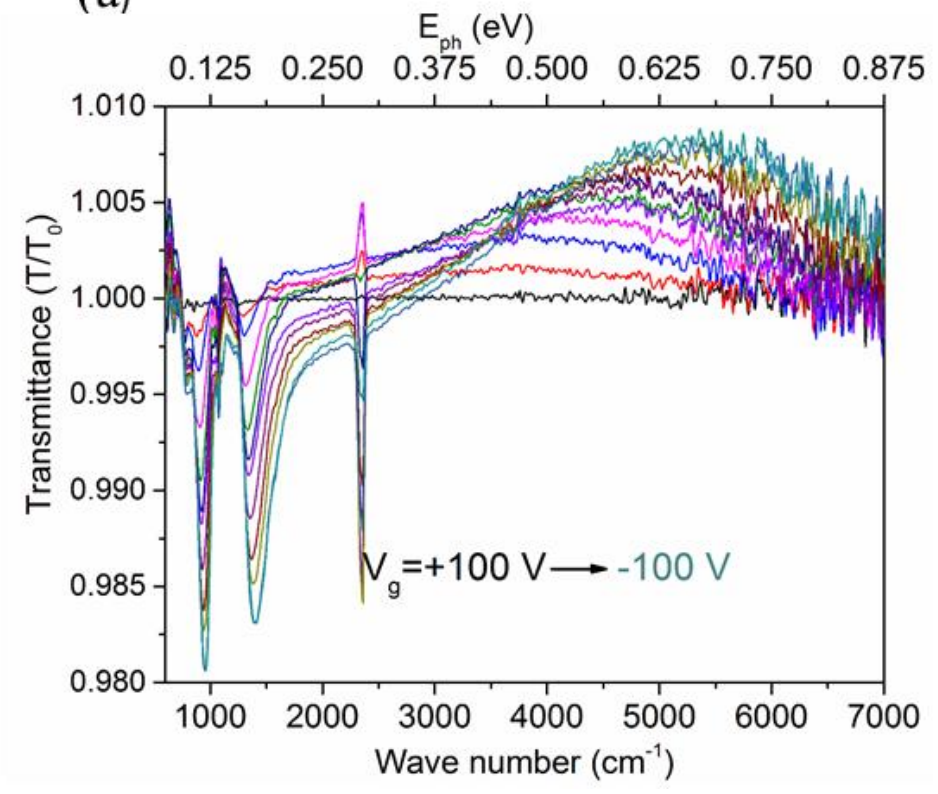

(b)

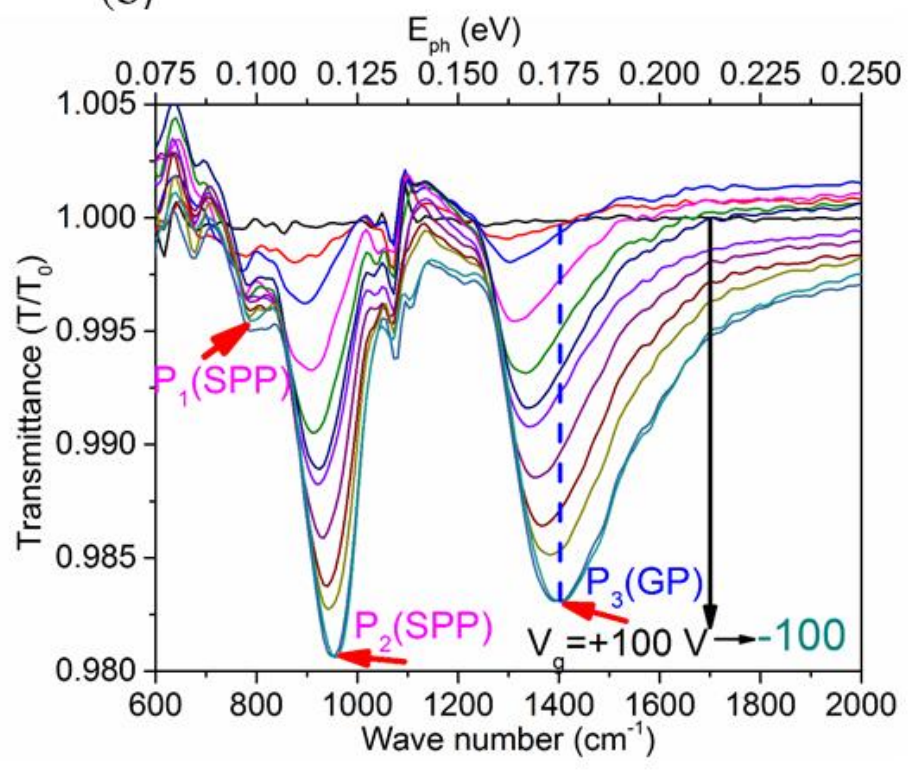

FIG.2. Gate dependent FTIR spectra normalized to spectra taken at $+100 \mathrm{~V} ; V_{g}$ varies from $+100 \mathrm{~V}$ to $-100 \mathrm{~V}$ in steps of $20 \mathrm{~V}$ in arrow direction. (a) FTIR transmission spectrum of $100 \mathrm{~nm}$ GNR array devices from 600- $7000 \mathrm{~cm}^{-1}$ (b) FTIR transmission spectrum of $100 \mathrm{~nm}$ from 600$2000 \mathrm{~cm}^{-1}$. 
(a)

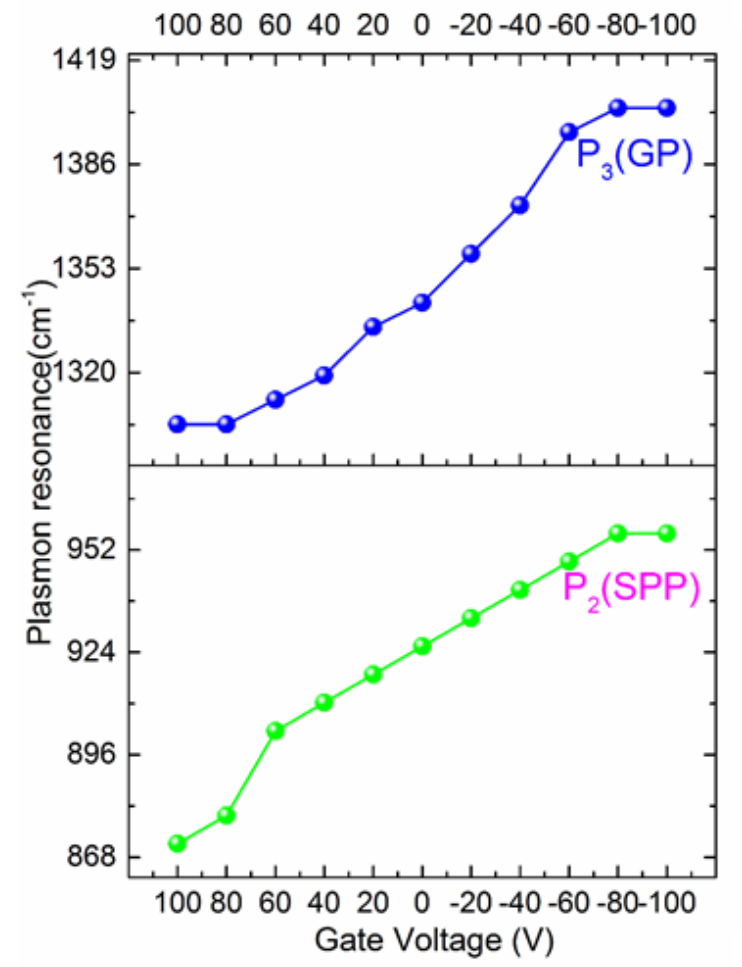

(b)

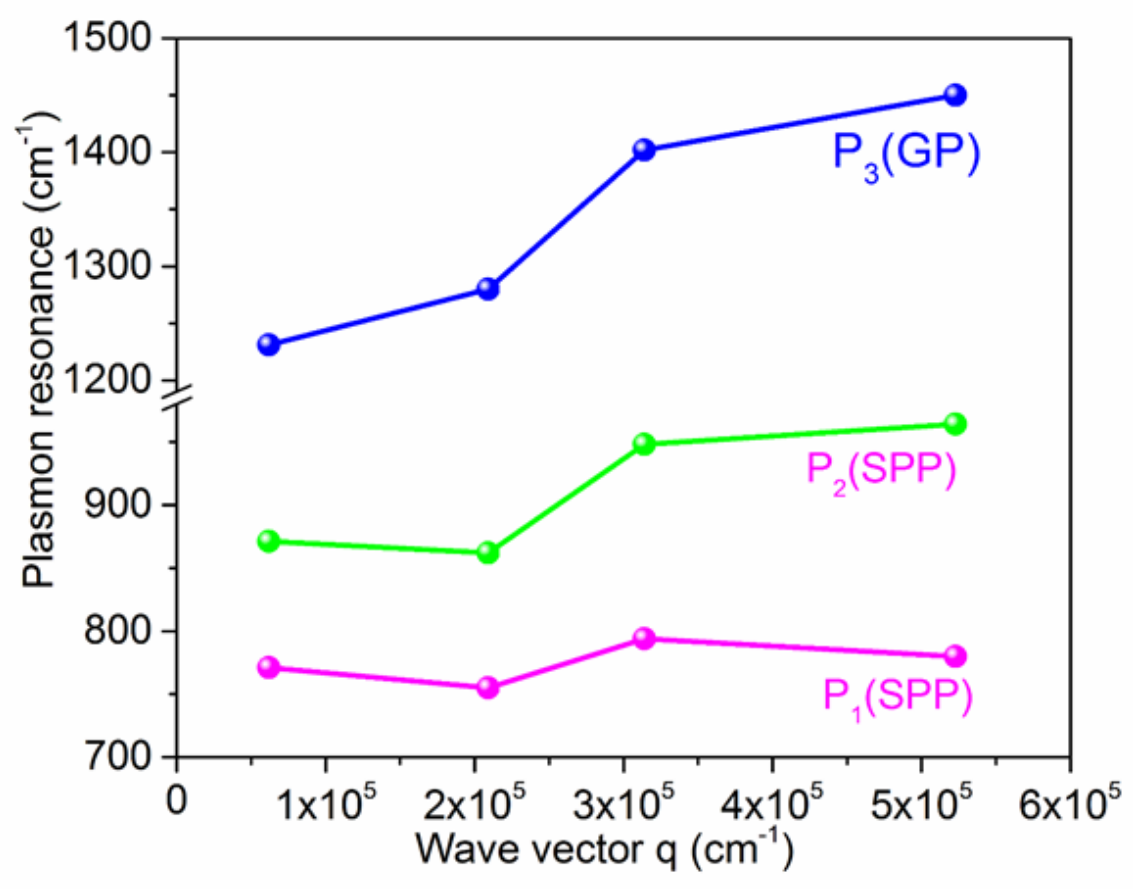

FIG.3. (a) Gate modulation of plasmon resonance peaks in $100 \mathrm{~nm}$ GNR array devices. (b) Plasmon frequency as a function of wave vector $q=\pi / W$ for plasmon resonance peaks 1,2 and 3 for GNR of width 60, 100, 150, and $500 \mathrm{~nm}$. 
(a)

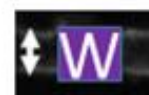

(c)

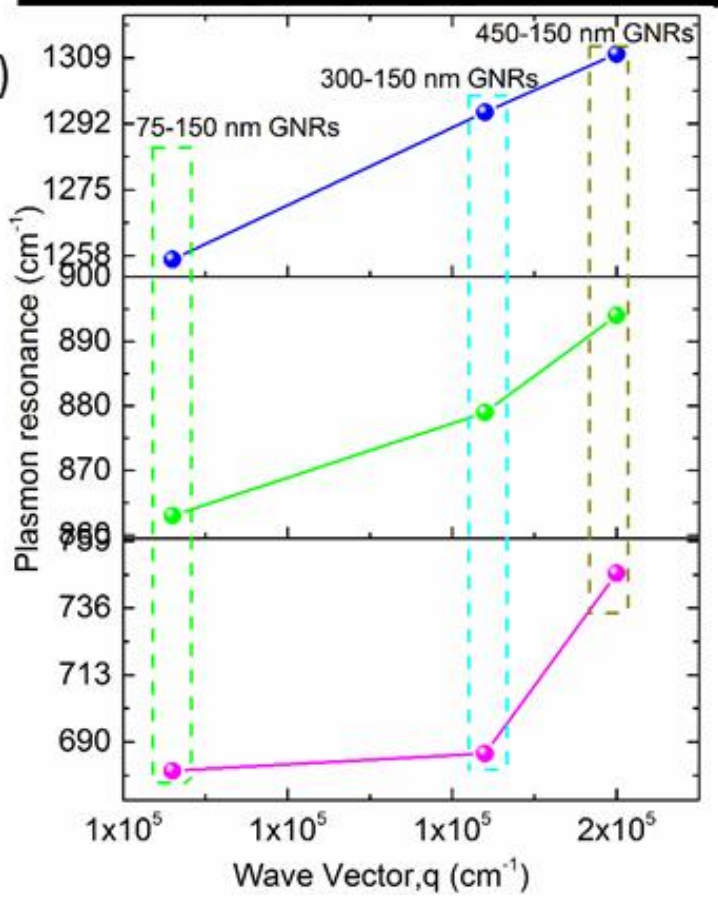

(b)

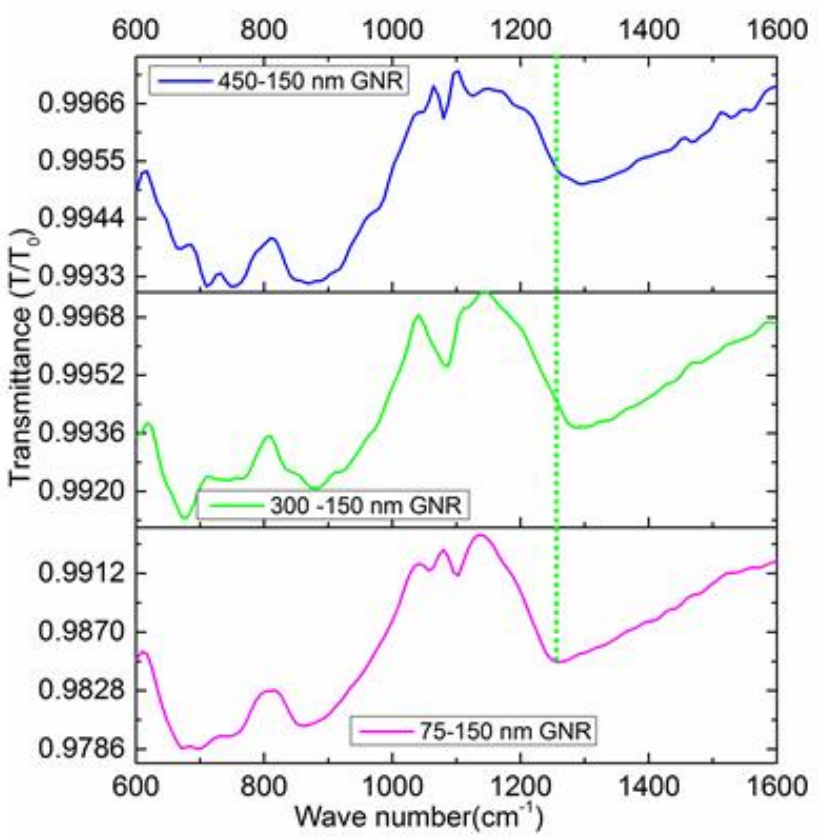

FIG.4. (a) A typical SEM image of a 450-150 nm GNR ( $W=150 \mathrm{~nm}, L=600 \mathrm{~nm}$; Aspect ratio $(L / W)=4)$. Gate dependent FTIR spectra normalized to spectra taken at $+100 \mathrm{~V}$ (b) Gate modulation of plasmon resonance peaks in GNR array devices with different aspect ratio (1.5-4), (c) Dispersion of plasmon resonance peaks in GNR array devices of aspect ratio (1.5-4). 